\title{
Some properties of cellular automata with equicontinuity points.
}

\author{
F. BLANCHARD and P. TISSEUR *
}

\begin{abstract}
We investigate topological and ergodic properties of cellular automata having equicontinuity points. In this class surjectivity on a transitive SFT implies existence of a dense set of periodic points. Our main result is that under the action of such an automaton any shift-ergodic measure converges in Cesàro mean, assuming equicontinuity points have measure 1 ; the limit measure is described by a formula and some of the properties of its topological support are given.
\end{abstract}

\section{Introduction.}

Compared with their topological dynamics, the ergodic theory of cellular automata is still in its infancy. One of the main reasons is that few invariant measures are known, if any, for any given cellular automaton. In this article for a family of CA defined by a topological property we give a rather simple construction of measures that are invariant both for the shift and the automaton; we also show that when an automaton belonging to this family is onto, there is a dense set of periodic points for its action.

The property of having equicontinuity points was first considered for cellular automata by Gilman [6] in relation with Wolfram's empirical classification [14]. Afterwards this property was used by Kưrka 8 as a basic element of his topological classification of CA according to their local behaviour. He distinguishes four classes: equicontinuous automata (E1); those that have equicontinuity points without being equicontinuous (E2); and two other classes of CA, all of them sensitive to initial conditions.

In 9] Lind finds the exact Cesàro mean limit of the images of Bernoulli measures by a simple additive cellular automaton. His result was generalized in [12] and later extended to Markov measures under the action of a larger class of additive cellular automata, all of them without equicontinuity points, by Ferrari, Maass, Martinez and Ney [5. Boyle and Kitchens [1] also proved that periodic points

\footnotetext{
*Institut de Mathématiques de Luminy - UPR 9016 du CNRS
} 
are dense for left- or right-closing automata; this class has only a small overlap with E2.

These results concern $\mathrm{CA}$ acting on the full set of configurations $A^{\mathbb{Z}}$. Our setting is slightly larger: we consider a cellular automaton $F$ acting on a subshift $X \subset A^{\mathbb{Z}}$, that is, $F(X) \subset X$, and the equicontinuity points we consider are those of the dynamical system $(X, F)$.

The article is devised as follows. After the Introduction, Section 2 is devoted to general definitions, in particular that of blocking words, which is essential for CA having equicontinuity points. Section 3 contains the results. The first one is topological but we prove it with the help of Poincaré's recurrence theorem: assuming that the automaton $F$ acts surjectively on a transitive subshift of finite type $X$ and has equicontinuity points, $X$ contains a dense set of $F$-periodic points. Our main result is purely ergodic: if a blocking word $(X, F)$ has positive measure for a shift-ergodic measure $\mu$, then the images of $\mu$ under the powers of $F$ converge in Cesàro mean. The limit $\mu_{c}$ is of course $F$ - and $\sigma$-invariant; it is given by a formula, which nevertheless leaves open several interesting questions. Then we examine properties of the topological support of $\mu_{c}$, and give several examples of cellular automata to which our results can be applied; we finish with some open questions.

\section{Definitions and background.}

\subsection{Dynamical systems, measures, cellular automata.}

A topological dynamical system $(X, T)$ consists of a compact metric space $X$ and a continuous self-map $T$.

A point $x \in X$ is said to be an equicontinuity point, or to be Lyapunov stable, if for any $\epsilon>0$, there exists $\eta>0$ such that if $d(x, y) \leq \eta$ one has $d\left(T^{i}(x), T^{i}(y)\right) \leq \epsilon$ for any integer $i>0$. When all points of $(X, T)$ are equicontinuity points $(X, T)$ is said to be equicontinuous: since $X$ is compact an equicontinuous system is uniformly equicontinuous.

The dynamical systems in this article are all defined on a symbolic space. Let $A$ be a finite alphabet. Define $A^{*}$ to be the set of all finite concatenations of letters of $A$, called words; the length of the word $u$ is denoted by $|u|$. A language $L$ is a subset of $A^{*}$.

The set $A^{\mathbb{Z}}$ of bi-infinite sequences on $A$ is endowed with the product topology, associated with the distance $d(x, y)=2^{-i}$ where $i=\min \{|j| x(j) \neq y(j)\}$; the shift $\sigma: \sigma(x)=\left(x_{i+1}\right)_{i \in \mathbb{Z}}$ is a homeomorphism on $A^{\mathbb{Z}}$. Given a word $u$ and an integer $t$, the clopen set $[u]_{t}=\left\{x \in A^{\mathbb{Z}}: x_{t}=u_{1} \ldots ; x_{t+|u|-1}=u_{|u|}\right\}$ is called a cylinder set. When $x \in A^{\mathbb{Z}}$ and $p \leq q$ are two integers, put $x(p, q)=x_{p} \ldots x_{q}$. A sequence $\left(S_{i}\right)_{i \in \mathbb{N}}$ is said to be ultimately periodic if there exist two natural integers $p^{\prime}$ and $p$ such that $S_{p^{\prime}+k p+i}=S_{p^{\prime}+i}$ for $k, i \in \mathbb{N}$.

The dynamical system $\left(A^{\mathbb{Z}}, \sigma\right)$ is called the full shift. A subshift $X$ is a closed shift-invariant subset of $A^{\mathbb{Z}}$. A transitive subshift $S$ is one such that for $u, v \in$ 
$L(S)$ there is $w \in L(S)$ such that $u w v \in L(S)$; it is strongly mixing if for any $n$ larger than some $n_{0}(u, v)$ one can find a word $w$ of length $n$ with the same property. To any subshift $X$ there corresponds a unique language $L(X)$ : it is the set of all words that are found as blocks of coordinates of a point of $X$. Given any subshift $X$ the language $L(X)$ has two general properties: for $u \in L(X)$, any sequence of consecutive letters of $u$ is also in $L(X)$; and for any word $v$ in $L(X)$ there are letters $a$ and $b$ in $A$ such that $a v b \in L(X))$. A subshift of finite type $X$ is defined by forbidding a finite family of words $E$ : then $L(X)$ is the smallest language having the two properties above and such that no word $u \in L(X)$ is of the form $u=v e w$ with $e \in E$. Transitive subshifts of finite type have a dense set of periodic points.

When a probability measure $\mu$ on $A^{\mathbb{Z}}$ is shift-invariant, its topological support $S(\mu)$ is closed invariant, hence a subshift. On every transitive subshift of finite type one defines a particular measure $\lambda$ with support $X$ called the Parry measure; the Parry measure of the full shift is the uniform measure.

A sequence $\left(\mu_{n}\right)_{n \in \mathbb{N}}$ of probability measures on a compact set $K$ is said to converge vaguely to a limit $\mu$ if the sequence $\int_{K} f d \mu_{n}$ tends to $\int_{K} f d \mu$ for any continuous function $f: K \rightarrow$. On a subshift $X$ a sequence $\left(\mu_{n}\right)_{n \in \mathbb{N}}$ of shiftinvariant measures converges vaguely if and only if for any word $u \in L(X)$ the sequence $\left(\mu_{n}\left([u]_{0}\right)\right)_{n \in \mathbb{N}}$ converges.

In this article we call cellular automaton(CA for short) a continuous map $F$ : $X \rightarrow X$ defined on a subshift $X \subseteq A^{\mathbb{Z}}$ and commuting with the shift $\sigma$; we also call cellular automatonthe dynamical system $(X, F)$. The Curtis-HedlundLyndon theorem [4] states that for every cellular automaton $(X, F)$ there is an integer $r$, called the radius of $F$, and a block map $f: A^{2 r+1} \cap L(X) \rightarrow A$ such that one has

$$
F(x)_{i}=f\left(x_{i-r}, \ldots, x_{i}, \ldots, x_{i+r}\right) .
$$

If $X$ is a transitive subshift of finite type, the automaton $F$ acts surjectively on $X$ if and only if the Parry measure $\lambda$ is $F$-invariant 3 .

The set $W(X, F)=\lim _{n \rightarrow \infty} \bigcap_{i=0}^{n} F^{i}(X)$ is called the limit set of the cellular automaton $(X, F)$; of course when $F$ is surjective $W(X, F)=X$.

\subsection{Blocking words and equicontinuous points.}

Definition: Let $F$ be a cellular automatonwith radius $r$ acting on the subshift $X$. A word $B \in A^{2 k+1}$ is called a blocking word for $(X, F)$ if there is an infinite sequence of words $v_{n},\left|v_{n}\right|=2 i+1 \geq r$ such that for any $x \in A^{\mathbb{Z}}$ with $x(-k, k)=B$ one has $F^{n}(x)(-i, i)=v_{n}$ for $n \in \mathbb{Z}^{*}$.

In the definition above we do not assume $X$ to be $\sigma$-transitive, but this condition appears necessary for most of our proofs. Remark that if $B$ is a blocking word and $x(-k, k)=B$, then $F^{n}(x)(-\infty,-i)$ does not depend on $x(k,+\infty)$, and reversely since $2 i+1 \geq r$. An occurrence of a blocking word in a configuration $x$ completely disconnects coordinates to its right and left for the action of the automaton; hence the name. 
The two following results are essentially due to Kůrka 8 .

Proposition 2.1 Any equicontinuity point of a cellular automaton $(X, F)$ has an occurrence of a blocking word. Conversely if there exist blocking words, any point with infinitely many occurrences of a blocking word to the left and right is an equicontinuity point; if moreover $X$ is transitive for $\sigma$ equicontinuity points are dense in $X$.

Proof: Let $x$ be an equicontinuity point of $(X, F)$; applying the definition of equicontinuity points to cellular automata, there is an integer $k$ such that if $d(x, y)<2^{-k}$, for any $n$ one has $F^{n} x(-r, r)=F^{n} y(-r, r)$, so that $B=$ $x(-k, k)$ is a blocking word. Conversely let $V(X)$ be the set of all points with infinitely many occurrences of blocking words to the left and right; when $X$ is transitive $V(X)$ is non-empty, even dense. Let $x \in V(X)$. To any given $\varepsilon>0$ one associates an integer $t$ such that $2^{-t}<\varepsilon$. There exist a real number $\eta$ and integers $t<k$ such that $2^{-k}<\eta$ and the words $x(-k,-t)$ and $x(t, k)$ contain an occurrence of $B$ each. For every point $y$ belonging to the cylinder set $[x(-k, k)]_{-k}$ one has $F^{i}(x)(-t, t)=F^{i}(y)(-t, t)$; since $\varepsilon$ is chosen arbitrarily one concludes that $x$ is an equicontinuity point.

For an equicontinuous cellular automaton, there is a natural integer $k$ such that all words of $L(X)$ with length $2 k+1$ are blocking words; thus

Proposition 2.2 The cellular automaton $(X, F)$ is equicontinuous if and only if there are two integers $p$ and $p^{\prime}$ such that for $x \in X$ the sequence $\left(F^{n}(x)\right)_{n \in \mathbb{N}}$ is ultimately periodic with period $p$ and preperiod $p^{\prime}$.

\section{Results.}

\subsection{Dense periodic points.}

Proposition 3.1 Let $X$ be a transitive subshift of finite type and suppose that the cellular automaton $(X, F)$ has an equicontinuity point. Then $F$ is onto if and only if it possesses a dense set of periodic points.

Proof: Let $F$ act surjectively on the transitive subshift of finite type $X$ and suppose it has equicontinuity points. For any word $v \in L(X)$ we construct a $\sigma$-periodic point $\bar{u} \in X$ such that $\bar{u}(k,|v|-1+k)=v$ for some integer $k$, which is also $F$-periodic; this establishes the density of $F$-periodic points in $X$.

Fix $v \in L(X)$. By Proposition $2.1 F$ has a blocking word $B \in L(X)$; as $X$ is transitive and has a dense set of $\sigma$-periodic points, there is a word $u=$ $B w v w^{\prime} \in L(X)$ such that $\bar{u} \in X$, where $\bar{u}$ is the periodic point constructed on $u$ and such that an occurrence of $u$ starts at 0 . The cylinder set $C=[u B]_{0}$ contains $\bar{u}$, and $\lambda(C)>0$ if $\lambda$ is the Parry measure of $X$. Since $\lambda$ is $F-$ invariant we apply the Poincaré recurrence theorem: there is $m>0$ such that $\lambda\left(C \cap F^{-m} C\right)>0$; in particular there are a point $x \in X$ and $q=(|u B|-1)$ such that $x(0, q)=F^{m}(x)(0, q)=u B$. 
But $B$ is a blocking word. All the coordinates of $\bar{u}$ coincide with those of $x$ on the segment $[0, q]$, and since there is an occurrence of $B$ at the beginning of this segment and one at the end, for any $n>0$ one has $F^{n} x(i, q-i)=F^{n} \bar{u}(i, q-i)$, where $i<\frac{1}{2}|B|$ is as in the definition of blocking words. We have thus shown that $F^{n} x$ and $F^{n} \bar{u}$ coincide on a segment of length $q-2 i \geq q-|B|=|u|$, which is greater than or equal to the common $\sigma$-period of $\bar{u}$ and $F^{m} \bar{u}$ : therefore $F^{m} \bar{u}=\bar{u}$.

The converse is straightforward.

We have proved this topological result ergodically. There should be a purely combinatorial proof. The following simple consequence is known but seems to be nowhere in written form.

Corollary 3.1 A cellular automaton $(X, F)$ is equicontinous and surjective if and only if there is $p>0$ such that any $x \in X$ is periodic of period $p$.

Proof:

By Proposition 2.2 $F$ being equicontinuous, there is an integer $p^{\prime}$ such that for any $x \in A^{\mathbb{Z}}$ the sequence $\left(F^{p^{\prime}+n}(x)\right)_{n \in \mathbb{N}}$ is periodic with period $p$; then any periodic point has period $p$. By Proposition 3.1 the set of periodic points is dense; as in the proof of this proposition one identifies the block of coordinates $F^{n}(x)(-k, k)$ with the corresponding block of a periodic point with period $p$ for every $n$, and one reaches the conclusion by letting $k$ go to infinity.

\subsection{Cesàro mean convergence of measures.}

We start with an easy result on equicontinuous CA. If $\mu$ is a measure on $A^{\mathbb{Z}}$ and $M$ a Borel set, denote by

$$
\mu_{n}(M)=\frac{1}{n} \sum_{i=0}^{n-1} \mu\left(F^{-i}(M)\right)
$$

its Cesàro mean of order $n$ with respect to $F$.

Proposition 3.2 Let $(X, F)$ be an equicontinous cellular automatonwith period $p$ and preperiod $p^{\prime}$, and let $\mu$ be a shift-ergodic measure with support $X$. Then $\mu$ converges vaguely in Cesàro mean to the measure $\mu_{c}=\frac{1}{p} \sum_{i=0}^{p-1} \mu \circ F^{-\left(i+p^{\prime}\right)}$.

Proof:

It suffices to show that for $u \in L(X)$ the sequence $\left(\mu_{n}\left([u]_{0}\right)\right)_{n \in \mathbb{N}}$ converges to the right limit. By Proposition 2.2 there are $p$ and $p^{\prime}$ that for any point $x$, any pair of integers $n$ and $i$ one has $F^{p^{\prime}+i+n p}(x)=F^{p^{\prime}+i}(x)$. Thus if $u \in L(X)$ and $n>p^{\prime}$ one has

$$
\mu_{n}\left([u]_{0}\right)=\frac{1}{n} \sum_{i=0}^{p^{\prime}-1} \mu\left(F^{-i}\left([u]_{0}\right)\right)+\frac{1}{n} \sum_{i=p^{\prime}}^{n-1} \mu\left(F^{-i}\left([u]_{0}\right)\right) .
$$


The first term tends to 0 ; using periodicity one gets

$$
\mu_{n}\left([u]_{0}\right)=\frac{1}{p} \sum_{i=0}^{p-1} \mu\left(F^{-\left(i+p^{\prime}\right)}\left([u]_{-k}\right)\right) .
$$

Definition: Let $F$ be a cellular automatonacting on the subshift $X$. A probability measure $\mu$ on $X$ is said to be equicontinuous for $(X, F)$ if the set of equicontinuity points of $(X, F)$ has measure 1 .

Lemma 3.1 Let $(X, F)$ be a cellular automatonand $\mu$ be a measure on $X$, ergodic for $\sigma$. Then the two following properties are equivalent:

- (1) there exists a blocking word $B$ such that $\mu\left([B]_{0}\right)>0$;

- (2) $\mu$ is equicontinuous for $(X, F)$.

Proof: $\quad(1) \Rightarrow(2)$ : since $\mu$ is $\sigma$-ergodic and $\mu\left([B]_{0}\right)>0$, almost every point contains infinitely many occurrences of $B$ to the left and right, so it is an equicontinuity point by Proposition 2.1

$(2) \Rightarrow(1)$ : again by Proposition 2.1] every equicontinuity point contains an occurrence of a blocking word; since the family of blocking words is at most countable, there is a blocking word $B$ such that $\mu\left([B]_{0}\right)>0$.

Definition: Given a word $B$, which we shall always suppose to be a blocking word for $(X, F)$, let $R(k, m, B)$ be the set of all points of $X$ having at least one occurrence of $B$ between the coordinates $-m-k$ and $-k$, and another one between the coordinates $k$ and $m+k$. Whenever there is no ambiguity on $B$ we denote it by $R(k, m)$.

Theorem 3.1 Let $(X, F)$ be a cellular automatonand $\mu$ be a shift-ergodic, equicontinuous measure on $X$. Then $\mu$ converges vaguely in Cesàro mean under $F$. The limit $\mu_{c}$ is $F$ - and $\sigma$-invariant, and for every word $u \in L(X)$ one has

$$
\mu_{c}\left([u]_{0}\right)=\lim _{m \rightarrow \infty} \frac{1}{p(k, m)} \sum_{i=0}^{p(k, m)-1} \mu\left(R(k, m) \cap F^{-(i+\bar{p}(k, m))}\left([u]_{0}\right)\right) .
$$

Proof: It is sufficient to show that for any word $w \in L(X),|w|=2 k+1$, the sequence $\left(\mu_{n}\left([w]_{-k}\right)\right)_{n \in \mathbb{N}}$ converges.

By Lemma 3.1 there is a blocking word $B$ for $(X, F)$ with $\mu\left([B]_{0}\right)>0$. The limit of the increasing sequence of sets $(R(k, m))_{m \in \mathbb{N}}$ is the set of all points having at least two occurrences of $B$, one to the left of $-k$ and the other to the right of $k$. Since $\mu$ is $\sigma$-ergodic the set $V(B)$ of points having infinitely many occurrences 
of $B$ to the right and left has measure 1. Thus $\lim _{m \rightarrow \infty} \mu(R(k, m))=1$ and for any integer $k$, any word $w \in L(X) \cap A^{2 k+1}$ one has

$$
\mu_{n}\left([w]_{-k}\right)=\lim _{m \rightarrow \infty} \frac{1}{n} \sum_{i=0}^{n-1} \mu\left(F^{-i}\left([w]_{-k}\right) \cap R(k, m)\right) .
$$

We prove that $\mu_{n}\left([w]_{-k}\right)$ by using the twofold convergence of the double sequence $\left(\frac{1}{n} \sum_{i=0}^{n-1} \mu\left(F^{-i}\left([u]_{-k}\right) \cap R(k, m)\right)\right)_{m, n \in \mathbb{N}}$. Indeed since the interval $[0,1]$ in which $\mu$ takes its values is compact, if $\left(\frac{1}{n} \sum_{i=0}^{n-1} \mu\left(F^{-i}\left([u]_{-k}\right) \cap R(k, m)\right)\right)_{m, n \in \mathbb{N}}$ converges simply as $n \rightarrow \infty$ and uniformly in $n$ as $m \rightarrow \infty$, the two limits commute and one obtains the desired convergence:

$$
\lim _{n \rightarrow \infty} \lim _{m \rightarrow \infty} \frac{1}{n} \sum_{i=0}^{n-1} \mu\left(F^{-i}\left([u]_{-k}\right) \cap R(k, m)\right)=\lim _{n \rightarrow \infty} \mu_{n}\left([w]_{-k}\right) .
$$

Let us show first that the sequence converges as $n \rightarrow \infty$ for fixed $m$.

Let $x$ and $y$ belong to $R(k, m)$ : by the definition of $R(k, m)$ there are blocking words to the left of their $-k^{t h}$ coordinate and to the right of their $k^{t h}$ coordinate, so that if $y$ is such that $u=y(-m-k, m+k)=x(-m-k, m+k)$, then for any integer $i$ one has $F^{i}(x)(-k, k)=F^{i}(y)(-k, k)$. In particular if $\bar{u}$ is the periodic point with period $2 m+2 k+1$ such that $\bar{u}(-m-k, m+k)=u$, the sequence $\left(F^{n}(\bar{u})\right)_{n \in \mathbb{N}}=\left(F^{n}(x)(-k, k)\right)_{n \in \mathbb{N}}$ is ultimately periodic. Let $p(x, k, m)$ be its period and $p^{\prime}(x, k, m)$ be its preperiod. Denote by $p(k, m)$ the least common multiple of the values of $p(x, k, m)$ for $x \in R(k, m)$ and by $p^{\prime}(k, m)$ the corresponding integer for $p^{\prime}(x, k, m)$.

Let $w$ be a word of length $2(k+m)+1$ such that $[w]_{-k-m} \subset R(k, m)$. For any $x \in[w]_{-k-m}$ and $i, j \in \mathbb{N}$ one has $F^{p^{\prime}(k, m)+j+i p(k, m)}(x)(-k, k)=$ $F^{p^{\prime}(k, m)+j}(x)(-k, k)$; thus for any word $u$ of length $2 k+1$ one has

$$
R(k, m) \cap F^{-\left(i p(k, m)+j+p^{\prime}(k, m)\right.}\left([u]_{-k}\right)=R(k, m) \cap F^{-\left(p^{\prime}(k, m)+j\right)}\left([u]_{-k}\right) .
$$

An argument similar to that of the proof of Proposition 3.2 shows that

$$
\begin{aligned}
\lim _{n \rightarrow \infty} \frac{1}{n} \sum_{i=0}^{n-1} \mu\left(F^{-i}\left([u]_{-k}\right) \cap R(k, m)\right) \\
\quad=\frac{1}{p(k, m)} \sum_{i=0}^{p(k, m)-1} \mu\left(F^{-(i+\bar{p}(k, m))}\left([u]_{-k}\right) \cap R(k, m)\right)
\end{aligned}
$$

which is what we want. Now let us prove that the sequence

$$
\left.\left(\frac{1}{n} \sum_{i=0}^{n-1} \mu(R(k, m)) \cap F^{-i}\left([u]_{-k}\right)\right)\right)_{m \in \mathbb{N}}
$$

converges uniformly in $n$ when $m \rightarrow \infty$. 
We already know that for any real number $\varepsilon>0$, for fixed $k$ there is an integer $m_{0}$ such that whenever $m \geq m_{0}$ one has $\mu(R(k, m)) \geq 1-\varepsilon$. Thus for any integer $i$ and $m \geq m_{0}$ one has

$$
\left|\mu\left((X-R(k, m)) \cap F^{-i}\left([u]_{-k}\right)\right)\right| \leq \varepsilon,
$$

hence

$$
\left|\mu\left(F^{-i}\left([u]_{-k}\right)\right)-\mu\left(R(k, m) \cap F^{-i}\left([u]_{-k}\right)\right)\right| \leq \varepsilon .
$$

For any integer $n$ if $m \geq m_{0}$ one has

$$
\left|\frac{1}{n} \sum_{i=0}^{n-1} \mu\left(R(k, m) \cap F^{-i}\left([u]_{-k}\right)\right)-\frac{1}{n} \sum_{i=0}^{n-1} \mu\left(F^{-i}\left([u]_{-k}\right)\right)\right| \leq \frac{n \varepsilon}{n}=\varepsilon .
$$

Since the two convergence conditions hold, we have proved that the two following limits exist and are the same:

$$
\begin{gathered}
\mu_{c}\left([u]_{-k}\right)=\lim _{n \rightarrow \infty} \lim _{m \rightarrow \infty} \frac{1}{n} \sum_{i=0}^{n-1} \mu\left(R(k, m) \cap F^{-i}\left([u]_{-k}\right)\right) \\
=\lim _{m \rightarrow \infty} \lim _{n \rightarrow \infty} \frac{1}{n} \sum_{i=0}^{n-1} \mu\left(R(k, m) \cap F^{-i}\left([u]_{-k}\right)\right)=\lim _{n \rightarrow \infty} \mu_{n}\left([u]_{-k}\right) .
\end{gathered}
$$

Equality (1) permits to conclude that

$$
\mu_{c}\left([u]_{-k}\right)=\lim _{m \rightarrow \infty} \frac{1}{p(k, m)} \sum_{i=0}^{p(k, m)-1} \mu\left(R(k, m) \cap F^{-(i+\bar{p}(k, m))}\left([u]_{-k}\right)\right) .
$$

The next corollary generalizes Theorem 3.1 to a larger class of cellular automata. Its proof is straightforward.

Corollary 3.2 Let $(X, F, \mu)$ and $k \in \mathbb{Z}$ be such that $\mu$ is $\sigma$-ergodic and equicontinuous for $\left(X, F \circ \sigma^{-k}\right)$; then the conclusions of Theorem 3.1 hold.

\subsection{The topological support of the measure $\mu_{c}$.}

Remark first that the topological support $S\left(\mu_{c}\right)$ is contained in $W(F)$.

Recall that $R(k, m)$ is the set of points with at least one occurrence of $B$ between the coordinates $-k-m$ and $-m$ and another one between the coordinates $m$ and $m+k$. We start with a technical lemma.

Lemma 3.2 Let $\mu$ be a $\sigma$-ergodic measure, equicontinuous for $(X, F)$, and let $B$ be a blocking word such that $\mu\left([B]_{0}\right)>0$. For any word $u$ in $A^{2 k+1}$ the sequence

$$
W_{m}(u)=\frac{1}{p(k, m)} \sum_{i=0}^{p(k, m)-1} \mu\left(R(k, m) \cap F^{-\left(i+p^{\prime}(k, m)\right)}\left([u]_{-k}\right)\right)
$$


is non-decreasing.

Proof: Let $m_{1}<m_{2}$ be two natural integers. The two sequences $\left(U_{i}\right)=$ $\left(\mu\left(R\left(k, m_{2}\right) \cap F^{-i}\left([u]_{-k}\right)\right)\right)$ and $\left(V_{i}\right)=\left(\mu\left(R\left(k, m_{1}\right) \cap F^{-i}\left([u]_{-k}\right)\right)\right)$ are ultimately periodic with preperiod and period $p^{\prime}\left(k, m_{2}\right)$ and $p\left(k, m_{2}\right)$ for the former, $p^{\prime}\left(k, m_{1}\right)$ and $p\left(k, m_{1}\right)$ for the latter.

Denote by $p^{\prime}$ the greatest of the two integers $p^{\prime}\left(k, m_{2}\right)$ and $p^{\prime}\left(k, m_{1}\right)$, and put $p=p\left(k, m_{2}\right) \times p\left(k, m_{1}\right)$. The sequences $\left(V_{i}\right)$ and $\left(U_{i}\right)$ are ultimately periodic with preperiod $p^{\prime}$ and period $p$ so that

$$
W_{m_{2}}(u)=\frac{1}{p} \sum_{i=0}^{p-1} \mu\left(R\left(k, m_{2}\right) \cap F^{-\left(i+p^{\prime}\right)}\left([u]_{-k}\right) .\right.
$$

Since $R\left(k, m_{1}\right) \subset R\left(k, m_{2}\right)$ one has

$$
\begin{gathered}
W_{m_{2}}(u) \geq \frac{1}{p} \sum_{i=0}^{p-1} \mu\left(R\left(k, m_{1}\right) \cap F^{-\left(i+p^{\prime}\right)}\left([u]_{-k}\right)\right. \\
=\frac{1}{p\left(k, m_{1}\right)} \sum_{i=0}^{p\left(k, m_{1}\right)-1} \mu\left(R\left(k, m_{1}\right) \cap F^{-\left(i+p^{\prime}\left(k, m_{1}\right)\right)}\left([u]_{-k}\right)=W_{m_{1}}(u) .\right.
\end{gathered}
$$

Proposition 3.3 Suppose $X$ is a transitive subshift of finite type, $F$ is onto and $\mu$ is equicontinuous for $(X, F)$ and $\sigma$-ergodic; then $S\left(\mu_{c}\right) \supset S(\mu)$.

Proof:

Choose a blocking word $B$ such that $\mu\left([B]_{0}\right)>0$, and $y \in S(\mu)$; since $\mu\left([y(-k, k)]_{-k}\right)>0$ for any integer $k$ and $\lim _{m \rightarrow \infty} \mu(R(k, m))=1$, there is an integer $m_{0}$ such that whenever $m \geq m_{0}$ one has $\mu\left([y(-k, k)]_{-k} \cap R(k, m)\right)>0$. For $m \in \mathbb{N}$ choose a point $x_{m}$ in $[y(-k, k)]_{-k} \cap R(k, m)$. By Proposition 3.1 the set of $F$-periodic points is dense so there exists one, $y_{m}$, in the cylinder set $\left[x_{m}(-k-m, k+m)\right]_{-k-m}$. The sequence $\left(F^{n}\left(x_{m}\right)(-k, k)\right)$ does not depend on the coordinates to the left of $-k-m$ and to the right of $k+m$; it is identical to the periodic sequence $F^{n}\left(y_{m}\right)(-k, k)$; in particular $p^{\prime}(k, m)=0$.

Fix $k$ and $m$ : the sequence of sets $\left(F^{-i}\left([y(-k, k)]_{-k}\right) \cap R(k, m)\right)_{i \geq 0}$ is periodic; since $\mu\left([y(-k, k)]_{-k} \cap R(k, m)\right)>0$ one has, in the notation of Lemma 3.2

$$
\begin{gathered}
W_{m}(y(-k, k))=\frac{1}{p(k, m)} \sum_{i=0}^{p(k, m)-1} \mu\left(R(k, m) \cap F^{-i}\left([y(-k, k)]_{-k}\right) \geq\right. \\
\frac{1}{p(k, m)} \mu\left((R(k, m)) \cap[y(-k, k)]_{-k}\right)>0 .
\end{gathered}
$$

By Proposition 3.1 and Lemma 3.2 the sequence $W_{m}(y(-k, k))$ is nondecreasing and tends to $\mu_{c}\left([y(-k, k)]_{-k}\right)$ so that $\mu_{c}\left([y(-k, k)]_{-k}\right)>0$ and finally $y \in S\left(\mu_{c}\right)$. 
In particular when $F$ is onto and $S(\mu)=A^{\mathbb{Z}}$ one has $S\left(\mu_{c}\right)=A^{\mathbb{Z}}$.

Let $(X, F)$ be a cellular automatonhaving equicontinuity points. For any blocking word $B$, call $E(F, B)$ the set of all points $y \in X$ such that for any natural integer $k$, there is another natural integer $m_{0}$ such that $\forall m \geq m_{0}$ and $\forall i \geq p^{\prime}(k, m)$ one has $[y(-k, k)]_{-k} \cap F^{i}(R(k, m)) \neq \emptyset$.

Proposition 3.4 The set $E(F, B)$ is a subshift; one has $F(E(F, B)) \subset$ $E(F, B)$, thus $E(F, B)$ is contained in the limit set $W(F)$; if $F$ is surjective, $E(F, B)=A^{\mathbb{Z}}$. If $X$ is transitive (resp. strongly mixing) for $\sigma$, then $E(F, B)$ does not depend on the choice of the word $B$ and can be denoted by $E(F)$; it is also transitive (resp. strongly mixing) for $\sigma$.

Proof: Since the definition of $E(F, B)$ depends only on the blocks of coordinates of its points, $E(F)$ is a subshift; the fact that $F(E(F, B)) \subset E(F, B)$ derives from the same remark. Let $X$ be transitive, $B$ and $B^{\prime}$ be two arbitrary blocking words; if $[y(-k, k)]_{-k} \cap F^{i}(R(k, m, B)) \neq \emptyset$, then $[y(-k, k)]_{-k} \cap$ $F^{i}\left(R(k, m, B) \cap F^{i}\left(R\left(k, m^{\prime}, B^{\prime}\right)\right) \neq \emptyset\right.$ provided $m^{\prime}$ is big enough, which implies $E(F, B) \subset E\left(F, B^{\prime}\right)$. Transitivity or strong mixing result from the fact that two words in $L(E(F))$ can occur in the image under $F^{n}$ of one point containing one or several blocking words between their respective occurrences.

Proposition 3.5 If $\mu$ is equicontinuous for $(X, F), S\left(\mu_{c}\right) \subset E(F)$. If moreover $S(\mu)=X$, then $S\left(\mu_{c}\right)=E(F)$.

Proof: Fix $B$ and assume that $y \notin E(F)$, so there is an integer $k$ such that for any integer $m$ and for any integer $i \geq p^{\prime}(k, m)$ one has $[y(-k, k)]_{-k} \not \subset$ $F^{i}(R(k, m))$. Thus

$$
\frac{1}{p(k, m)} \sum_{i=0}^{p(k, m)-1} \mu\left(R(k, m) \cap F^{-\left(i+p^{\prime}(k, m)\right)}\left([y(-k, k)]_{-k}\right)\right)=0 .
$$

Applying Proposition 3.1 one obtains $\mu_{c}\left([y(-k, k)]_{-k}\right)=0$ and $y \notin S\left(\mu_{c}\right)$. Hence $S\left(\mu_{c}\right) \subset E(F)$.

If $S(\mu)=X$ it is sufficient to prove that $S\left(\mu_{c}\right) \supset E(F)$. If $x \in E(F)$, for any natural integer $k$ there is $m \in \mathbb{N}$ such that the union of cylinder sets $\left.G=F^{-p^{\prime}(k, m)}\left([x(-k, k)]_{-k}\right) \cap R(k, m)\right)$ is not empty. Hence $\mu(G)>0$. By Lemma 3.2 and Proposition 3.1 the sequence indexed by $m$

$$
\frac{1}{p(k, m)} \sum_{i=0}^{p(k, m)-1} \mu\left(R(k, m) \cap F^{-\left(i+p^{\prime}(k, m)\right)}\left([y(-k, k)]_{-k}\right)\right)
$$

is non-decreasing and since $\mu(G)>0$, Proposition 3.1 implies that $\mu_{c}$ $\left([x(-k, k)]_{-k}\right)>0$ and finally $x \in S\left(\mu_{c}\right)$.

Examples

In [10], [8], 2] one can find examples of cellular automata that are surjective on 
$A^{\mathbb{Z}}$ and have equicontinuity points. One can therefore apply Proposition 3.1 and also Theorem 3.1 and Proposition 3.3 if one assumes that $\mu$ is for instance a Bernoulli measure $B\left(p_{1}, p_{2}, p_{3}\right)$ different from the uniform measure. In [13] the automaton called "Gliders and walls" has equicontinuity points without being onto.

Here is another example: the cellular automaton $F:\{0,1,2\}^{\mathbb{Z}} \rightarrow\{0,1,2\}^{\mathbb{Z}}$ with radius 1 is defined by the local map $f$ such that $f\left(x_{-1}, x_{0}, 2\right)=x_{0}$, $f\left(x_{-1}, 2, x_{1}\right)=2$ and when $x_{1} \in\{0,1\}$ and $x_{0} \neq 2$ then $f\left(x_{-1}, x_{0}, x_{1}\right)=$ $x_{0}+x_{1} \bmod 2 . F$ is onto; it has equicontinuity points since 2 is a blocking word. Let $\mu$ be a Bernoulli measure with parameters $\{p, q, r\}$ on $A^{\mathbb{Z}}$; by considering the cylinder sets $[2012]_{0}$ and $[2112]_{0}$ one easily checks that the sequence $\mu \circ F^{n}$ does not converge vaguely. By Theorem 3.1 it converges in Cesàro mean but, still considering the same two cylinder sets, the limit cannot be the Bernoulli measure with parameters $\left\{\frac{p+q}{2}, \frac{p+q}{2}, r\right\}$.

\section{Questions}

When is $\mu_{c}$ ergodic for $F$ ? When $F$ is onto and $\mu$ is the uniform measure, which is $F$-invariant in this case, $\mu_{c}=\mu$ is never $F$-ergodic (this would imply transitivity of $F$, which in its turn implies sensitivity).

When is it $\sigma$-ergodic?

Are there conditions for $\mu_{c}$ to be the uniform measure, or at least Bernoulli or Markov?

\section{Acknowledgements}

We want to thank A. Maass for many suggestions; we are also grateful to the referee, who signalled various shortcomings. Part of this work was done by the second author at Universidad de Chile in Santiago, thanks to the financial support of Fondap-Modelamiento Estocastico and Ecos-Conicyt.

\section{References}

[1] M. Boyle, B. Kitchens, Periodic points in cellular automata, prétirage (1999). À paraître, Indagationes Math.

[2] F. Blanchard, A. MaAss, Dynamical behaviour of Coven's aperiodic cellular automata, Theoret. Computer Sci. 163 (1996), 291-302.

[3] E. Coven, M. Paul, Endomorphisms of irreducible shifts of finite type, Math. Sys. Th. 8 (1974), 167-175.

[4] M. Denker, C. Grillenberger, K. Sigmund, Ergodic theory on compact spaces. Lecture Notes in Math. 527, Springer, Berlin (1975).

[5] P. Ferrari, A. MaAss, S. Martínez, P. Ney, Cesàro mean distribution of group automata starting from measures with summable decay, preprint (1999).

[6] R. H. Gilman, Classes of linear automata, Ergodic Th. \& Dynam. Sys. 7 (1987), 105-118. 
[7] G. A. Heduund, Endomorphisms and automorphisms of the shift dynamical system, Math. Systems Th. 3 (1969), 320-375.

[8] P. KŮRKA, Languages, equicontinuity and attractors in linear cellular automata, Ergod. Th. \& Dynam. Sys. 217 (1997), 417-433..

[9] D. A. Lind, Applications of ergodic theory and sofic systems to cellular automata, Physica 10D (1984), 36-44.

[10] D. A. Lind, Entropies of automorphisms of a topological Markov shift, Proc. Amer.Math.Soc. 99 (1987), 589-595.

[11] A. MaAss, On sofic limit sets of cellular automata, Ergodic Th. Dynam. \& Sys. 15 (1995), 663-684.

[12] A. Maass, S. Martínez, On Cesàro limit distribution of a classof permutative cellular automata, J. Statist. Physics 90 (1998), 435-452.

[13] J. Milnor, On the entropy geometry of cellular automata, Complex Systems 2 (1988), 357-386.

[14] S. Wolfram, Theory and applications of cellular automata, World Scientific, 1986. 